\title{
GORENSTEIN PROJECTIVE, INJECTIVE AND FLAT MODULES
}

\author{
ZHONGKUI LIU and XIAOYAN YANG ${ }^{凶}$
}

\author{
(Received 28 February 2008; accepted 26 December 2008)
}

Communicated by J. Du

\begin{abstract}
In basic homological algebra, projective, injective and flat modules play an important and fundamental role. In this paper, we discuss some properties of Gorenstein projective, injective and flat modules and study some connections between Gorenstein injective and Gorenstein flat modules. We also investigate some connections between Gorenstein projective, injective and flat modules under change of rings.
\end{abstract}

2000 Mathematics subject classification: primary 16E30; secondary 16E65.

Keywords and phrases: Gorenstein projective module, Gorenstein injective module, Gorenstein flat module, change of ring.

\section{Introduction}

Unless stated otherwise, throughout this paper all rings are associative with identity, and all modules are unitary. Let $R$ be a ring. We denote by $R$-Mod and Mod- $R$ the categories of left and right $R$-modules, respectively. For any $R$-module $M, \operatorname{pd}_{R} M$, $\operatorname{id}_{R} M$, and $\operatorname{fd}_{R} M$ denote the projective, injective, and flat dimensions respectively. The character module $\operatorname{Hom}_{Z}(M, Q / Z)$ is denoted by $M^{+}$.

When $R$ is two-sided Noetherian, Auslander and Bridger [2] introduced the Gdimension, G- $\operatorname{dim}_{R} M$, for every finitely generated $R$-module $M$. They proved that $\mathrm{G}-\operatorname{dim}_{R} M \leq \operatorname{pd}_{R} M$ with equality $\mathrm{G}-\operatorname{dim}_{R} M=\mathrm{pd}_{R} M$ when $\operatorname{pd}_{R} M$ is finite. Over a general ring $R$, Enochs and Jenda defined in [6] a homological dimension, namely the Gorenstein projective dimension $\operatorname{Gpd}_{R}(-)$, for arbitrary (nonfinite) modules. It is defined via resolution with (so-called) Gorenstein projective modules. Avramov, Bachweitz, Martsinkovsky and Reiten proved that a finite module over a Noetherian ring is Gorenstein projective if and only if $\mathrm{G}-\operatorname{dim}_{R} M=0$ (see the remark

Research supported by National Natural Science Foundation of China, TRAPOYT and the Cultivation Fund of Key Scientific and Technical Innovation Project, Ministry of Education of China.

(c) 2009 Australian Mathematical Publishing Association, Inc. 1446-7887/2009 \$16.00 
following [3, Theorem 4.2.6]). Holm [9] gave homological descriptions of the Gorenstein dimensions over arbitrary rings. He proved that these dimensions are similar to the classical homological dimensions; that is, the projective, injective and flat dimensions.

In Section 2, we discuss some properties of Gorenstein projective, injective and flat modules and we also discuss connections between Gorenstein injective and flat modules. In Section 3, we investigate some connections between Gorenstein projective, injective and flat modules under change of rings. We shall then be concerned with what happens when certain modifications are made to a ring.

We first recall some concepts. Let $\mathcal{X}$ be any class of $R$-modules, $M$ an $R$-module. A left $\mathcal{X}$-resolution of $M$ is an exact sequence $\mathbb{X}: \cdots \rightarrow X_{1} \rightarrow X_{0} \rightarrow M \rightarrow 0$ with $X_{i} \in \mathcal{X}$ for all $i \geq 0$. A right $\mathcal{X}$-resolution of $M$ is an exact sequence $\mathbb{X}: 0 \rightarrow M \rightarrow$ $X^{0} \rightarrow X^{1} \rightarrow \cdots$ with $X^{i} \in \mathcal{X}$ for all $i \geq 0$. Now let $\mathbb{X}$ be any (left or right) $\mathcal{X}$ resolution of $M$. We say that $\mathbb{X}$ is proper or co-proper when the sequence $\operatorname{Hom}_{R}(Y, \mathbb{X})$ or $\operatorname{Hom}_{R}(\mathbb{X}, Y)$ is exact for all $Y \in \mathcal{X}$ respectively.

A complete projective resolution is an exact sequence of projective modules

$$
\mathbb{P}: \cdots \longrightarrow P_{1} \longrightarrow P_{0} \longrightarrow P^{0} \longrightarrow P^{1} \longrightarrow \cdots
$$

such that $\operatorname{Hom}_{R}(\mathbb{P}, Q)$ is exact for every projective $R$-module $Q$. An $R$-module $M$ is called Gorenstein projective (G-projective for short) if there exists a complete projective resolution $\mathbb{P}$ with $M \cong \operatorname{Im}\left(P_{0} \rightarrow P^{0}\right)$. Every projective module is Gorenstein projective. The class of all Gorenstein projective $R$-modules is denoted by $\mathcal{G P}(R)$. Holm [9] proved that the class $\mathcal{G P}(R)$ is closed under arbitrary direct sums and under direct summands. Gorenstein injective (G-injective for short) modules are defined dually and every injective module is Gorenstein injective. The class of all such modules is denoted by $\mathcal{G I}(R)$. Holm [9] proved that the class $\mathcal{G I}(R)$ is closed under any direct products and under direct summands. A complete flat resolution is an exact sequence of flat (left) modules

$$
\mathbb{F}: \cdots \longrightarrow F_{1} \longrightarrow F_{0} \longrightarrow F^{0} \longrightarrow F^{1} \longrightarrow \cdots
$$

such that $I \otimes_{R} \mathbb{F}$ is exact for every injective right $R$-module $I$. An $R$-module $M$ is called Gorenstein flat (G-flat for short) if there exists a complete flat resolution $\mathbb{F}$ with $M \cong \operatorname{Im}\left(F_{0} \rightarrow F^{0}\right)$. Every flat module is Gorenstein flat. The class of all Gorenstein flat $R$-modules is denoted by $\mathcal{G F}(R)$. Holm [9] proved that the class $\mathcal{G} \mathcal{F}(R)$ is closed under arbitrary direct sums.

\section{Properties of Gorenstein modules}

It is well known that $R$ is a perfect ring if and only if any direct limit of projective $R$-modules is projective by [16, Theorem 1.2.13].

THEOREM 2.1. Let $R$ be a left perfect, right coherent ring. If $M_{0} \rightarrow M_{1} \rightarrow M_{2} \rightarrow \cdots$ is a sequence of $G$-projective left $R$-modules, then the direct limit $\lim _{\longrightarrow} M_{n}$ is again G-projective. 
PROOF. For each $n$, there exists a co-proper right projective resolution $\mathbb{P}_{n}: 0 \rightarrow$ $M_{n} \rightarrow P_{n}^{0} \rightarrow P_{n}^{1} \rightarrow \cdots$. Consider the following commutative diagram:

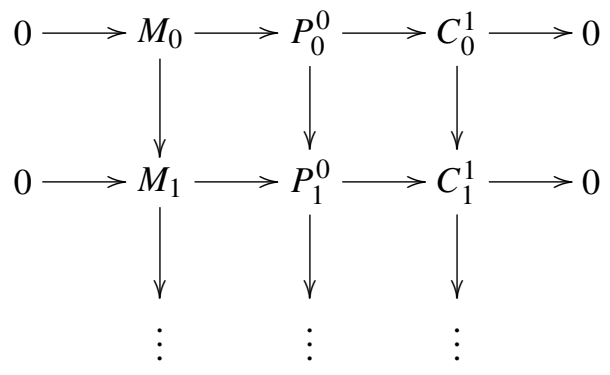

where $C_{n}^{1}=\operatorname{Coker}\left(M_{n} \rightarrow P_{n}^{0}\right)$ for each $n$. Then each column in the above diagram is again a direct system. Thus $0 \rightarrow \underline{\lim } M_{n} \rightarrow \underset{\lim }{\longrightarrow} P_{n}^{0} \rightarrow \underline{\lim } C_{n}^{1} \rightarrow 0$ is exact by [7, Theorem 1.5.6], and $\lim _{\rightarrow} P_{n}^{0}$ is projective. Let $P$ be a projective left $R$-module. Then $\operatorname{Ext}_{R}^{1}\left(C_{n}^{1}, P\right)=0$, and so $C_{n}^{1}$ is G-projective by [9, Corollary 2.11] for each $n$. Let $Q$ be a projective left $R$-module. Then $Q$ is pure-injective by [16, Lemma 3.1.6]. Hence

$$
\begin{gathered}
\left.\operatorname{Ext}_{R}^{i} \stackrel{(\lim }{\longrightarrow} M_{n}, Q\right) \cong \underset{\lim }{\leftarrow} \operatorname{Ext}_{R}^{i}\left(M_{n}, Q\right)=0 \text { and } \\
\operatorname{Ext}_{R}^{i}\left(\stackrel{\lim }{\longrightarrow} C_{n}^{1}, Q\right) \cong \underset{\lim }{\leftarrow} \operatorname{Ext}_{R}^{i}\left(C_{n}^{1}, Q\right)=0
\end{gathered}
$$

by [15] for all $i \geq 1$, and so

$$
0 \rightarrow \operatorname{Hom}_{R}\left(\lim _{n} C_{n}^{1}, Q\right) \rightarrow \operatorname{Hom}_{R}\left(\lim _{\rightarrow} P_{n}^{0}, Q\right) \rightarrow \operatorname{Hom}_{R}\left(\stackrel{\lim }{\longrightarrow} M_{n}, Q\right) \rightarrow 0
$$

is exact. Continuing this procedure yields that $\operatorname{Hom}_{R}\left(\lim _{\rightarrow} \mathbb{P}_{n}, Q\right)$ is exact. It follows that $\underset{\lim }{\longrightarrow} M_{n}$ is G-projective.

It is well known that $R$ is a Noetherian ring if and only if any direct limit of injective $R$-modules is injective by [7, Theorem 3.1.17].

THEOREM 2.2. Let $R$ be left Artinian and let the injective envelope of every simple left $R$-module be finitely generated. If $M_{0} \rightarrow M_{1} \rightarrow M_{2} \rightarrow \cdots$ is a sequence of $G$-injective left $R$-modules, then the direct limit $\lim _{\longrightarrow} M_{n}$ is again $G$-injective.

PROOF. For each $n$, there exists a proper left injective resolution $\mathbb{E}_{n}: \cdots \rightarrow E_{n}^{1} \rightarrow$ $E_{n}^{0} \rightarrow M_{n} \rightarrow 0$. Then

$$
\underset{\lim }{\longrightarrow} \mathbb{E}_{n}: \cdots \longrightarrow \lim _{\longrightarrow} E_{n}^{1} \longrightarrow \lim _{\longrightarrow} E_{n}^{0} \longrightarrow \underset{\lim }{\longrightarrow} M_{n} \longrightarrow 0
$$

is exact by analogy with the proof of Theorem 2.1, and $\underset{\longrightarrow}{\rightarrow} E_{n}^{k}$ is injective for $k=0,1, \ldots$ Let $J$ be any injective left $R$-module. Then $\vec{J}=\bigoplus_{\Lambda} J_{\alpha}$, where $J_{\alpha}$ is an injective envelope of some simple left $R$-module by [10, Theorem 6.6.4]. So

$$
\operatorname{Hom}_{R}\left(J, \lim _{\longrightarrow} \mathbb{E}_{n}\right) \cong \lim _{\Lambda} \prod_{\Lambda} \operatorname{Hom}_{R}\left(J_{\alpha}, \mathbb{E}_{n}\right)
$$


is exact by [7, Theorem 1.5.6] and

$$
\operatorname{Ext}_{R}^{i}\left(J, \lim _{\longrightarrow} M_{n}\right) \cong \lim _{\Lambda} \prod_{\Lambda} \operatorname{Ext}_{R}^{i}\left(J_{\alpha}, M_{n}\right)=0
$$

by [7, Theorem 3.1.16] for all $i \geq 1$. Therefore $\underset{\lim }{\longrightarrow} M_{n}$ is G-injective.

Holm in [9, Theorem 3.6] proved that if $R$ is right coherent, then $M$ is a G-flat left $R$-module if and only if $M^{+}$is a G-injective right $R$-module.

TheOREM 2.3. Let $R$ be left Artinian and let the injective envelope of every simple left $R$-module be finitely generated. Then the following are equivalent.

(1) $M$ is a G-injective left $R$-module.

(2) $M^{+}$is a $G$-flat right $R$-module.

PROOF. We show first that (1) implies (2). There exists a complete injective resolution $\mathbb{E}: \cdots \rightarrow E_{1} \rightarrow E_{0} \rightarrow E^{0} \rightarrow E^{1} \rightarrow \cdots$ with $M \cong \operatorname{Ker}\left(E^{0} \rightarrow E^{1}\right)$. Then

$$
\mathbb{E}^{+}: \cdots \longrightarrow E^{1+} \longrightarrow E^{0+} \longrightarrow E_{0}^{+} \longrightarrow E_{1}^{+} \longrightarrow \cdots
$$

is exact such that $M^{+} \cong \operatorname{Coker}\left(E^{1+} \rightarrow E^{0+}\right)$ and $E_{i}^{+}, E^{i+}$ are flat for $i=0,1, \ldots$ Let $J$ be any injective left $R$-module. Then $J=\bigoplus_{\Lambda} J_{\alpha}$, where $J_{\alpha}$ is an injective envelope of some simple left $R$-module by [10, Theorem 6.6.4], and so

$$
\mathbb{E}^{+} \otimes_{R} J \cong \bigoplus_{\Lambda}\left(\mathbb{E}^{+} \otimes_{R} J_{\alpha}\right) \cong \bigoplus_{\Lambda} \operatorname{Hom}_{R}\left(J_{\alpha}, \mathbb{E}\right)^{+}
$$

is exact by [7, Theorem 3.2.11]. It follows that $M^{+}$is a G-flat right $R$-module.

Now we prove that (2) implies (1). There exists a complete flat resolution $\mathbb{F}$ : $\cdots \rightarrow F_{1} \rightarrow F_{0} \rightarrow F^{0} \rightarrow F^{1} \rightarrow \cdots$ such that $\mathbb{F}_{l}: \cdots \rightarrow F_{1} \rightarrow F_{0} \rightarrow M^{+} \rightarrow 0$ and $\mathbb{F}_{r}: 0 \rightarrow M^{+} \rightarrow F^{0} \rightarrow F^{1} \rightarrow \cdots$ are exact. Then

$$
\begin{gathered}
\mathbb{F}_{r}^{+}: \cdots \longrightarrow F^{1+} \longrightarrow F^{0+} \longrightarrow M^{++} \longrightarrow 0 \text { and } \\
\quad \mathbb{F}_{l}^{+}: 0 \longrightarrow M^{++} \longrightarrow F_{0}^{+} \longrightarrow F_{1}^{+} \longrightarrow \cdots
\end{gathered}
$$

are exact. We successively pick injective left $R$-modules $E_{0}, E_{1}, \ldots$ and $E^{0}, E^{1}, \ldots$ such that

$$
\begin{gathered}
F_{0}^{+} \oplus E_{0} \cong F_{0}^{+++}, \quad F_{i}^{+} \oplus E_{i-1} \oplus E_{i} \cong\left(F_{i}^{+} \oplus E_{i-1}\right)^{++} \\
F^{0+} \oplus E^{0} \cong F^{0+++}, \quad F^{i+} \oplus E^{i-1} \oplus E^{i} \cong\left(F^{i+} \oplus E^{i-1}\right)^{++}
\end{gathered}
$$

for $i=1,2, \ldots$ By adding $0 \rightarrow E_{i} \rightarrow E_{i} \rightarrow 0$ to the sequence $\mathbb{F}_{l}^{+}$in degree $i+1$, $i+2$ and adding $0 \rightarrow E^{i} \rightarrow E^{i} \rightarrow 0$ to the sequence $\mathbb{F}_{r}^{+}$in degree $i+2, i+1$ for $i=0,1, \ldots$, we obtain the exact sequence

$$
\cdots \longrightarrow\left(F^{1+} \oplus E^{0}\right)^{++} \longrightarrow F^{0+++} \longrightarrow F_{0}^{+++} \longrightarrow\left(F_{1}^{+} \oplus E_{0}\right)^{++} \longrightarrow \cdots .
$$

So $\mathbb{E}: \cdots \rightarrow F^{1+} \oplus E^{0} \rightarrow F^{0+} \rightarrow F_{0}^{+} \rightarrow F_{1}^{+} \oplus E_{0} \rightarrow \cdots$ is exact such that $M \cong$ $\operatorname{Ker}\left(F_{0}^{+} \rightarrow F_{1}^{+} \oplus E_{0}\right)$ and $F^{0+}, F^{i+} \oplus E^{i-1}, F_{0}^{+}, F_{i}^{+} \oplus E_{i-1}$ are injective left $R$-modules for $i=1,2, \ldots$ Let $J$ be any injective left $R$-module. Then 
$J=\bigoplus_{\Lambda} J_{\alpha}$, where $J_{\alpha}$ is an injective envelope of some simple left $R$-module by [10, Theorem 6.6.4]. Hence $\operatorname{Hom}_{R}\left(J_{\alpha}, \mathbb{F}^{+}\right) \cong\left(\mathbb{F} \otimes_{R} J_{\alpha}\right)^{+}$is exact, and so

$$
\operatorname{Hom}_{R}\left(J_{\alpha}, \mathbb{E}\right)^{++} \cong\left(\mathbb{E}^{+} \otimes_{R} J_{\alpha}\right)^{+} \cong \operatorname{Hom}_{R}\left(J_{\alpha}, \mathbb{E}^{++}\right)
$$

is exact by [7, Theorem 3.2.11], which implies that $\operatorname{Hom}_{R}(J, \mathbb{E}) \cong \prod_{\Lambda} \operatorname{Hom}_{R}\left(J_{\alpha}, \mathbb{E}\right)$ is exact. Thus $M$ is a $\mathrm{G}$-injective left $R$-module.

COROLlary 2.4. Let $R$ be left Artinian and let the injective envelope of every simple left $R$-module be finitely generated. Then $M^{+}$is right $G$-flat for any $G$-injective left $R$-module $M$ if and only if $N^{++}$is right $G$-flat for any $G$-flat right $R$-module $N$.

LEMMA 2.5. Let $R$ be left Artinian and let the injective envelope of every simple left $R$-module be finitely generated. Then the class of all $G$-flat right $R$-modules is closed under arbitrary direct products.

Proof. Let $M=\prod_{i \in I} M_{i}$ with each $M_{i}$ a G-flat right $R$-module. Then there is a complete flat resolution $\mathbb{F}_{i}: \cdots \rightarrow F_{1 i} \rightarrow F_{0 i} \rightarrow F_{i}^{0} \rightarrow F_{i}^{1} \rightarrow \cdots$ with $M_{i} \cong$ $\operatorname{Ker}\left(F_{i}^{0} \rightarrow F_{i}^{1}\right)$ for each $i \in I$. Then

$$
\prod_{i \in I} \mathbb{F}_{i}: \cdots \longrightarrow \prod_{i \in I} F_{1 i} \longrightarrow \prod_{i \in I} F_{0 i} \longrightarrow \prod_{i \in I} F_{i}^{0} \rightarrow \prod_{i \in I} F_{i}^{1} \longrightarrow \cdots
$$

is exact such that $M \cong \operatorname{Ker}\left(\prod_{i \in I} F_{i}^{0} \rightarrow \prod_{i \in I} F_{i}^{1}\right)$ and $\prod_{i \in I} F_{i}^{k}, \prod_{i \in I} F_{k i}$ are flat for $k=0,1, \ldots$ Let $E$ be any injective left $R$-module. Then $E=\bigoplus_{\Lambda} E_{\alpha}$, where $E_{\alpha}$ is an injective envelope of some simple left $R$-module by [10, Theorem 6.6.4], and hence $\left(\prod_{i \in I} \mathbb{F}_{i}\right) \otimes_{R} E \cong \bigoplus_{\Lambda} \prod_{i \in I}\left(\mathbb{F}_{i} \otimes_{R} E_{\alpha}\right)$ is exact. So $M$ is a G-flat right $R$ module.

COROLlary 2.6. Let $R$ be left Artinian and let the injective envelope of every simple left $R$-module be finitely generated. Then the following are equivalent for an $(R, S)$ bimodule $M$.

(1) $M$ is a $G$-injective left $R$-module.

(2) $\operatorname{Hom}_{S}(M, E)$ is a $G$-flat right $R$-module for all injective right $S$-modules $E$.

(3) $\operatorname{Hom}_{S}(M, E)$ is a $G$-flat right $R$-module for any injective cogenerator $E$ for Mod-S.

(4) $\quad M \otimes_{S} F$ is a $G$-injective left $R$-module for all flat left $S$-modules $F$.

(5) $\quad M \otimes_{S} F$ is a $G$-injective left $R$-module for any faithfully flat left $S$-module $F$.

Proof. We show first that (1) implies (2). Let $E$ be any injective right $S$-module. Then $E$ is isomorphic to a summand of $S^{+X}$ for some set $X$, and so $\operatorname{Hom}_{S}(M, E)$ is isomorphic to a summand of $\operatorname{Hom}_{S}\left(M, S^{+X}\right) \cong M^{+X}$. It follows that $\operatorname{Hom}_{S}(M, E)$ is a G-flat right $R$-module by Theorem 2.3 and Lemma 2.5 .

That (2) implies (3) is obvious.

We prove that (3) implies (1). Since $M^{+} \cong \operatorname{Hom}_{S}\left(M, S^{+}\right)$is a G-flat right $R$ module, we have $M$ is a G-injective left $R$-module by Theorem 2.3. 
To show that (2) implies (4), let $F$ be any flat left $S$-module. Then $\left(M \otimes_{S} F\right)^{+} \cong$ $\operatorname{Hom}_{S}\left(M, F^{+}\right)$is a G-flat right $R$-module, and so $M \otimes_{S} F$ is a G-injective left $R$ module by Theorem 2.3.

That (4) implies (5) and (5) implies (1) are obvious.

A ring $R$ is said to be left V-ring if every simple left $R$-module is injective. Recall an $R$-module $M$ is small projective if $\operatorname{Hom}_{R}(M,-)$ is exact with respect to the exact sequence $0 \rightarrow K \rightarrow L \rightarrow M \rightarrow 0$ in $R$-Mod with $K \ll L$.

Corollary 2.7. Let $R$ be a left Artinian left V-ring ring. Then the following are equivalent for an $(R, S)$-bimodule $M$.

(1) $M$ is a G-injective left $R$-module.

(2) $\operatorname{Hom}_{S}(M, E)$ is a $G$-flat right $R$-module for all injective right $S$-modules $E$.

(3) $\operatorname{Hom}_{S}(M, E)$ is a $G$-flat right $R$-module for any injective cogenerator $E$ for Mod-S.

(4) $\quad M \otimes_{S} F$ is a $G$-injective left $R$-module for all flat left $S$-modules $F$.

(5) $\quad M \otimes_{S} F$ is a $G$-injective left $R$-module for any faithfully flat left $S$-module $F$.

Corollary 2.8. Let $R$ be a commutative Artinian ring. Then the following are equivalent for an $(R, S)$-bimodule $M$.

(1) $M$ is a $G$-injective $R$-module.

(2) $\operatorname{Hom}_{S}(M, E)$ is a $G$-flat $R$-module for all injective right $S$-modules $E$.

(3) $\operatorname{Hom}_{S}(M, E)$ is a $G$-flat $R$-module for any injective cogenerator $E$ for Mod- $S$.

(4) $\quad M \otimes_{S} F$ is a $G$-injective $R$-module for all flat left $S$-modules $F$.

(5) $M \otimes_{S} F$ is a $G$-injective $R$-module for any faithfully flat left $S$-module $F$.

Proof. If $L$ is a simple $R$-module, then $E(L)$ is finitely generated by [11, Theorem 3.64].

COROllary 2.9. Let $R$ be left Artinian. If every left $R$-module is small projective, then the following are equivalent for an $(R, S)$-bimodule $M$.

(1) $M$ is a G-injective left $R$-module.

(2) $\operatorname{Hom}_{S}(M, E)$ is a $G$-flat right $R$-module for all injective right $S$-modules $E$.

(3) $\operatorname{Hom}_{S}(M, E)$ is a $G$-flat right $R$-module for any injective cogenerator $E$ for Mod- $S$.

(4) $\quad M \otimes_{S} F$ is a $G$-injective left $R$-module for all flat left $S$-modules $F$.

(5) $\quad M \otimes_{S} F$ is a $G$-injective left $R$-module for any faithfully flat left $S$-module $F$.

An $R$-module $C$ is said to be cotorsion if $\operatorname{Ext}_{R}^{1}(F, C)=0$ for all flat $R$ modules $F$. It is well known that every module has a cotorsion envelope and if $\varphi: M \rightarrow C$ is a cotorsion envelope of an $R$-module $M$, then $L=\operatorname{Coker} \varphi$ is flat. An $R$-module $M$ is called FP-injective if $\operatorname{Ext}_{R}^{1}(N, M)=0$ for all finitely presented modules $N$. The FP-injective dimension of $M$, denoted by FP-id $M$, is defined to be the least nonnegative integer $n$ such that $\operatorname{Ext}_{R}^{n+1}(N, M)=0$ for all finitely presented modules $N$. If no such $n$ exists, set FP-id $M=\infty$. 
THEOREM 2.10. Let $R$ be two-sided coherent. Then the following are equivalent.

(1) $R$ is two-sided FP-injective.

(2) Every $R$-module (left and right) is G-flat.

(3) Every finitely presented $R$-module (left and right) is $G$-flat.

(4) Every finitely presented $R$-module (left and right) is G-projective.

(5) Every cyclic R-module (left and right) is G-flat.

(6) Every of the quotient module $R / P$ ( $P$ is a prime ideal) is G-flat.

(7) Every cotorsion $R$-module (left and right) is $G$-flat.

(8) Every nonzero $R$-module (left and right) contains a nonzero G-flat submodule.

PROOF. The equivalences (1) if and only if (2) if and only if (3) if and only if (4) hold by [5, Theorem 6].

The implications (1) implies (5), (1) implies (6) and (1) implies (7) are trivial.

We show that (6) implies (3). We use the fact that every finitely presented $R$-module $M$ can be filtered as

$$
M=M_{0} \supset M_{1} \supset \cdots \supset M_{k}=0,
$$

where successive quotients are isomorphic to $R / P$ for some prime ideal $P$. Hence $M_{k-1}$ is G-flat since $M_{k-2} / M_{k-1}$ is G-flat. Proceeding thus we can show that $M$ is G-flat.

To show that (7) implies (1), let $M$ be any $R$-module. Then there exists an exact sequence $0 \rightarrow M \rightarrow C(M) \rightarrow L \rightarrow 0$, where $C(M)$ is cotorsion and $L$ is flat, and so $M$ is G-flat by [9, Theorem 3.7].

To prove that (5) implies (8), let $M$ be any nonzero left or right $R$-module. Then there is $0 \neq x \in M$, and so $R x$ or $x R$ is a nonzero G-flat submodule of $M$ respectively.

We prove that (8) implies (2). Let $M$ be any nonzero $R$-module. Then there is a nonzero G-flat submodule $N$ of $M$. Set $\mathfrak{D}=\{N \subseteq D \subseteq M \mid D \in \mathcal{G F}(R)\}$. Then $\mathfrak{D}$ is a nonempty subposet of the lattice of submodules of $M$ and every nonempty chain in $\mathfrak{D}$ has an upper bound in $\mathfrak{D}$, namely its union. So by Zorn's Lemma, $\mathfrak{D}$ has a maximal element say $L$. If $L \neq M$, then $M / L \neq 0$, and so there is a nonzero G-flat submodule $K / L$ of $M / L$. Hence $K$ is G-flat by [9, Theorem 3.7] and $K$ is strictly larger than $L$, which gives that $M=L$ is G-flat.

Proposition 2.11. Let $R$ be a commutative ring $Q$ a flat $R$-module. If $M$ is a $G$-flat $R$-module, then $M \otimes_{R} Q$ is a $G$-flat $R$-module.

Proof. Since $M$ is G-flat, there exists a complete flat resolution $\mathbb{F}: \cdots \rightarrow F_{1} \rightarrow$ $F_{0} \rightarrow F^{0} \rightarrow F^{1} \rightarrow \cdots$ with $M \cong \operatorname{Ker}\left(F^{0} \rightarrow F^{1}\right)$. Then

$$
\mathbb{F} \otimes_{R} Q: \cdots \longrightarrow F_{1} \otimes_{R} Q \longrightarrow F_{0} \otimes_{R} Q \longrightarrow F^{0} \otimes_{R} Q \longrightarrow F^{1} \otimes_{R} Q \longrightarrow \cdots
$$

is exact such that $M \otimes_{R} Q \cong \operatorname{Ker}\left(F^{0} \otimes_{R} Q \rightarrow F^{1} \otimes_{R} Q\right)$ and $F_{i} \otimes_{R} Q, F^{i} \otimes_{R} Q$ are flat for $i=0,1, \ldots$ Let $E$ be any injective $R$-module. Then $E \otimes_{R}\left(\mathbb{F} \otimes_{R} Q\right)$ is exact, and so $M \otimes_{R} Q$ is a G-flat $R$-module.

PROPOSITION 2.12. Let $R$ be a commutative ring $P$ a finitely generated projective $R$-module. If $M$ is a $G$-flat $R$-module, then $\operatorname{Hom}_{R}(P, M)$ is a $G$-flat $R$-module. 
Proof. Let $Q$ be any flat $R$-module and let $0 \rightarrow A \rightarrow B$ be exact. Consider the commutative diagram

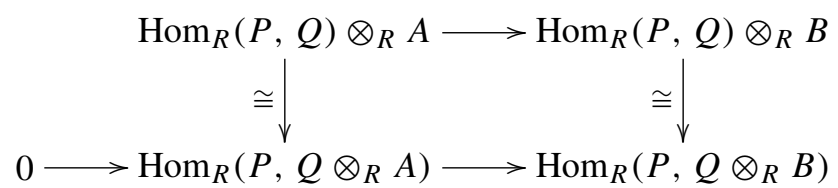

with the lower row exact. Then $\operatorname{Hom}_{R}(P, Q)$ is flat. Since $M$ is G-flat, there is a complete flat resolution $\mathbb{F}: \cdots \rightarrow F_{1} \rightarrow F_{0} \rightarrow F^{0} \rightarrow F^{1} \rightarrow \cdots$ with $M \cong$ $\operatorname{Ker}\left(F^{0} \rightarrow F^{1}\right)$. Then

$$
\begin{aligned}
& \operatorname{Hom}_{R}(P, \mathbb{F}): \cdots \longrightarrow \operatorname{Hom}_{R}\left(P, F_{1}\right) \longrightarrow \operatorname{Hom}_{R}\left(P, F_{0}\right) \longrightarrow \operatorname{Hom}_{R}\left(P, F^{0}\right) \\
& \quad \longrightarrow \operatorname{Hom}_{R}\left(P, F^{1}\right) \longrightarrow \cdots
\end{aligned}
$$

is exact such that $\operatorname{Hom}_{R}(P, M) \cong \operatorname{Ker}\left(\operatorname{Hom}_{R}\left(P, F^{0}\right) \rightarrow \operatorname{Hom}_{R}\left(P, F^{1}\right)\right)$ and all the $\operatorname{Hom}_{R}\left(P, F_{i}\right)$ and $\operatorname{Hom}_{R}\left(P, F^{i}\right)$ are flat for $i=0,1, \ldots$ Let $I$ be any injective $R$-module. Then $\operatorname{Hom}_{R}(P, \mathbb{F}) \otimes_{R} I \cong \operatorname{Hom}_{R}\left(P, \mathbb{F} \otimes_{R} I\right)$ is exact by [1, Proposition 20.10], which means that $\operatorname{Hom}_{R}(P, M)$ is G-flat.

Proposition 2.13. Let $R$ be a commutative Artinian ring. If $M$ is a $G$-injective $R$-module, then $\operatorname{Hom}_{R}(E, M)$ is a $G$-flat $R$-module for any injective $R$-module $E$.

Proof. Let $E$ be any injective $R$-module. Then $E=\bigoplus_{\Lambda} E_{\alpha}$, where $E_{\alpha}$ is an injective envelope of some simple $R$-module by [10, Theorem 6.6.4]. Since $M$ is G-injective, there is a complete injective resolution $\mathbb{E}: \cdots \rightarrow E_{1} \rightarrow E_{0} \rightarrow E^{0} \rightarrow E^{1}$ $\rightarrow \cdots$ with $M \cong \operatorname{Ker}\left(E^{0} \rightarrow E^{1}\right)$. Then

$$
\begin{aligned}
& \operatorname{Hom}_{R}(E, \mathbb{E}): \cdots \longrightarrow \operatorname{Hom}_{R}\left(E, E_{1}\right) \longrightarrow \operatorname{Hom}_{R}\left(E, E_{0}\right) \longrightarrow \operatorname{Hom}_{R}\left(E, E^{0}\right) \\
& \quad \longrightarrow \operatorname{Hom}_{R}\left(E, E^{1}\right) \longrightarrow \cdots
\end{aligned}
$$

is exact such that $\operatorname{Hom}_{R}(E, M) \cong \operatorname{Ker}\left(\operatorname{Hom}_{R}\left(E, E^{0}\right) \rightarrow \operatorname{Hom}_{R}\left(E, E^{1}\right)\right)$ and all the $\operatorname{Hom}_{R}\left(E, E_{i}\right)$ and $\operatorname{Hom}_{R}\left(E, E^{i}\right)$ are flat for $i=0,1, \ldots$ Let $I$ be any injective $R$-module. Then $I=\bigoplus_{\Lambda^{\prime}} I_{\beta}$, where $I_{\beta}$ is an injective envelope of some simple $R$-module, and so

$$
\begin{aligned}
I \otimes_{R} \operatorname{Hom}_{R}(E, \mathbb{E}) & \cong \bigoplus_{\beta \in \Lambda^{\prime}} \operatorname{Hom}_{R}\left(\operatorname{Hom}_{R}\left(I_{\beta}, \bigoplus_{\alpha \in \Lambda} E_{\alpha}\right), \mathbb{E}\right) \\
& \cong \bigoplus_{\beta \in \Lambda^{\prime}} \prod_{\alpha \in \Lambda} \operatorname{Hom}_{R}\left(\operatorname{Hom}_{R}\left(I_{\beta}, E_{\alpha}\right), \mathbb{E}\right)
\end{aligned}
$$

is exact by [7, Theorem 3.2.11] since $I_{\beta}$ and $E_{\alpha}$ are finitely generated by [11, Theorem 3.64]. It follows that $\operatorname{Hom}_{R}(E, M)$ is G-flat.

\section{Gorenstein modules and change of rings}

Let $M$ be an $R$-module. Then a sequence of central elements $a_{1}, \ldots, a_{n}$ in an ideal $I$ of $R$ is called an $M$-sequence if $\left(a_{1}, \ldots, a_{n}\right) M \neq M$ and $a_{i}$ is not a zero-divisor on $M /\left(a_{1}, \ldots, a_{i-1}\right) M$ for $1 \leq i \leq n$. 
Lemma 3.1. Let a be a regular element $M$ an $R$-module. Then

$$
0 \longrightarrow M \stackrel{a}{\longrightarrow} M \longrightarrow M / a M \longrightarrow 0
$$

is exact if and only if

$$
0 \longrightarrow M \stackrel{a \otimes_{R} 1}{\longrightarrow} M \longrightarrow R / a \otimes_{R} M \longrightarrow 0
$$

is exact.

PROOF. By analogy with the proof of [17, Lemma 3.10].

LEMMA 3.2. Let $M$ be an $R$-module and let $I$ be generated by an $R$-and $M$-sequence $\left(a_{1}, \ldots, a_{n}\right)$. Then $\operatorname{Ext}_{R / I}^{i}(M / I M,-) \cong \operatorname{Ext}_{R}^{i}(M,-)$ for all $i \geq 1$.

PROOF. By analogy with the proof of [17, Lemma 3.11].

THEOREM 3.3. Let $M$ be an $R$-module and let $I$ be generated by an $R$-and $M$-sequence $\left(a_{1}, \ldots, a_{n}\right)$. If $M$ is a G-projective left $R$-module, then $M / I M$ is $a$ $G$-projective left $R / I$-module.

PROOF. Take a co-proper right projective resolution $\mathbb{P}: 0 \rightarrow M \rightarrow P^{0} \rightarrow P^{1} \rightarrow \cdots$. Since $0 \rightarrow R \stackrel{a_{1}}{\rightarrow} R \rightarrow R / a_{1} R \rightarrow 0$ is exact, $0 \rightarrow P^{i} \stackrel{a_{1}}{\rightarrow} P^{i} \rightarrow P^{i} / a_{1} P^{i} \rightarrow 0$ is exact for $i=0,1, \ldots$ Hence

$$
0 \longrightarrow \mathbb{P} \longrightarrow \mathbb{P} \longrightarrow R / a_{1} R \otimes_{R} \mathbb{P} \longrightarrow 0
$$

is exact, so $R / a_{1} R \otimes_{R} \mathbb{P}$ is exact. As $0 \rightarrow R / a_{1} R \stackrel{a_{2}}{\rightarrow} R / a_{1} R \rightarrow R /\left(a_{1}, a_{2}\right) R \rightarrow 0$ is exact, $0 \rightarrow P^{i} / a_{1} P^{i} \stackrel{a_{2}}{\rightarrow} P^{i} / a_{1} P^{i} \rightarrow P^{i} /\left(a_{1}, a_{2}\right) P^{i} \rightarrow 0$ is exact for $i=0,1, \ldots$ Thus

$$
0 \longrightarrow R / a_{1} R \otimes_{R} \mathbb{P} \longrightarrow R / a_{1} R \otimes_{R} \mathbb{P} \longrightarrow R /\left(a_{1}, a_{2}\right) R \otimes_{R} \mathbb{P} \longrightarrow 0
$$

is exact, and so $R /\left(a_{1}, a_{2}\right) R \otimes_{R} \mathbb{P}$ is exact. Continuing this procedure yields that

$$
R / I \otimes_{R} \mathbb{P}: 0 \longrightarrow M / I M \longrightarrow P^{0} / I P^{0} \longrightarrow P^{1} / I P^{1} \longrightarrow \cdots
$$

is exact and every $P^{i} / I P^{i}$ is a projective left $R / I$-module since $\operatorname{Ext}_{R / I}^{1}(P / I P,-) \cong$ $\operatorname{Ext}_{R}^{1}(P,-)=0$ by Lemma 3.2 for any projective left $R$-module $P$. Let $\bar{Q}$ be any projective left $R / I$-module. Then $\operatorname{pd}_{R} \bar{Q}=n$ by [13, Proposition 5.8], and so $\operatorname{Hom}_{R / I}\left(R / I \otimes_{R} \mathbb{P}, \bar{Q}\right) \cong \operatorname{Hom}_{R}(\mathbb{P}, \bar{Q})$ is exact and $\operatorname{Ext}_{R / I}^{i}(M / I M, \bar{Q}) \cong$ $\operatorname{Ext}_{R}^{i}(M, \bar{Q})=0$ by Lemma 3.2 for all $i \geq 1$. Thus $M / I M$ is a G-projective left $R / I$ module.

THEOREM 3.4. Let $M$ be an $R$-module and let $I$ be generated by an $R$-and $M$-sequence $\left(a_{1}, \ldots, a_{n}\right)$. If $M$ is a $G$-flat left $R$-module, then $M / I M$ is a $G$-flat left $R / I$-module. 
Proof. Since $M$ is G-flat, there exists a complete flat resolution $\mathbb{F}: \cdots \rightarrow F_{1} \rightarrow$ $F_{0} \rightarrow F^{0} \rightarrow F^{1} \rightarrow \cdots$ with $M \cong \operatorname{Ker}\left(F^{0} \rightarrow F^{1}\right)$. Then

$$
R / I \otimes_{R} \mathbb{F}: \cdots \longrightarrow F_{1} / I F_{1} \longrightarrow F_{0} / I F_{0} \longrightarrow F^{0} / I F^{0} \longrightarrow F^{1} / I F^{1} \longrightarrow \cdots
$$

is exact much as in the proof of Theorem 3.3 such that $M / I M \cong \operatorname{Ker}\left(F^{0} / I F^{0} \rightarrow\right.$ $\left.F^{1} / F^{1}\right)$ and $F_{i} / I F_{i}, F^{i} / I F^{i}$ are flat left $R / I$-modules for $i=0,1, \ldots$ since

$$
\operatorname{Tor}_{1}^{R / I}(-, F / I F)^{+} \cong \operatorname{Ext}_{R / I}^{1}\left(F / I F,-^{+}\right) \cong \operatorname{Ext}_{R}^{1}\left(F,-^{+}\right) \cong \operatorname{Tor}_{1}^{R}(-, F)^{+}=0
$$

by Lemma 3.2 for any flat left $R$-module $F$. Let $\bar{E}$ be any injective right $R / I$-module and let $0 \rightarrow A \rightarrow B$ be exact in Mod- $R$. Consider the following commutative diagram:

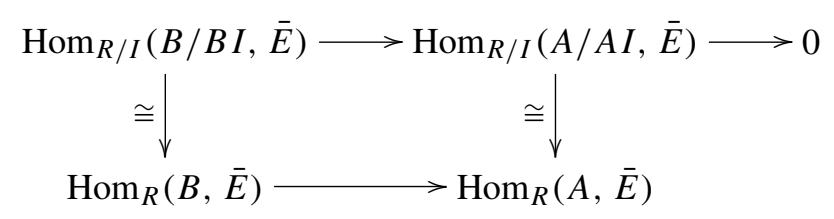

in which the upper row is exact. Then $\bar{E}$ is an injective right $R$-module. So $\bar{E} \otimes_{R / I}$ $\left(R / I \otimes_{R} \mathbb{F}\right) \cong \bar{E} \otimes_{R} \mathbb{F}$ is exact, which implies that $M / I M$ is a G-flat left $R / I$ module.

Theorem 3.5. Let $M$ be an $R$-module and let $I$ be generated by an $R$-and $M$ sequence $\left(a_{1}, \ldots, a_{n}\right)$. If $M$ is a $G$-injective right $R$-module, then $\operatorname{Hom}_{R}(R / I, M)$ is a $G$-injective right $R / I$-module.

Proof. Since $M$ is G-injective, there is a proper left injective resolution $\mathbb{E}$ : $\cdots \rightarrow E_{1} \rightarrow E_{0} \rightarrow M \rightarrow 0$. Since $0 \rightarrow R \rightarrow^{a_{1}} R \rightarrow R / a_{1} R \rightarrow 0$ is exact and $\operatorname{pd}_{R}\left(R / a_{1} R\right)=1$ by [11, Proposition 5.32],

$$
\begin{gathered}
0 \rightarrow \operatorname{Hom}_{R}\left(R / a_{1} R, M\right) \rightarrow M \rightarrow M \rightarrow 0 \text { and } \\
0 \rightarrow \operatorname{Hom}_{R}\left(R / a_{1} R, E_{i}\right) \rightarrow E_{i} \rightarrow E_{i} \rightarrow 0
\end{gathered}
$$

are exact by $[4$, Lemma 2.2] for $i=0,1, \ldots$ Thus

$$
0 \longrightarrow \operatorname{Hom}_{R}\left(R / a_{1} R, \mathbb{E}\right) \longrightarrow \mathbb{E} \longrightarrow \mathbb{E} \longrightarrow 0
$$

is exact, and so $\operatorname{Hom}_{R}\left(R / a_{1} R, \mathbb{E}\right)$ is exact. Since $0 \rightarrow R / a_{1} R \stackrel{a_{2}}{\rightarrow} R / a_{1} R \rightarrow$ $R /\left(a_{1}, a_{2}\right) R \rightarrow 0$ is exact and $\operatorname{pd}_{R}\left(R /\left(a_{1}, a_{2}\right) R\right)=2$ by [11, Proposition 5.32],

$$
\begin{aligned}
0 \longrightarrow \operatorname{Hom}_{R}\left(R /\left(a_{1}, a_{2}\right) R, M\right) & \longrightarrow \operatorname{Hom}_{R}\left(R / a_{1} R, M\right) \\
& \longrightarrow \operatorname{Hom}_{R}\left(R / a_{1} R, M\right) \longrightarrow 0 \\
0 \longrightarrow \operatorname{Hom}_{R}\left(R /\left(a_{1}, a_{2}\right) R, E_{i}\right) & \longrightarrow \operatorname{Hom}_{R}\left(R / a_{1} R, E_{i}\right) \\
& \longrightarrow \operatorname{Hom}_{R}\left(R / a_{1} R, E_{i}\right) \longrightarrow 0
\end{aligned}
$$


are exact by $[4$, Lemma 2.2] for $i=0,1, \ldots$ So

$0 \longrightarrow \operatorname{Hom}_{R}\left(R /\left(a_{1}, a_{2}\right) R, \mathbb{E}\right) \longrightarrow \operatorname{Hom}_{R}\left(R / a_{1} R, \mathbb{E}\right) \longrightarrow \operatorname{Hom}_{R}\left(R / a_{1} R, \mathbb{E}\right) \longrightarrow 0$

is exact, and hence $\operatorname{Hom}_{R}\left(R /\left(a_{1}, a_{2}\right) R, \mathbb{E}\right)$ is exact. Continuing this procedure yields

$$
\begin{aligned}
\operatorname{Hom}_{R}(R / I, \mathbb{E}): \cdots & \longrightarrow \operatorname{Hom}_{R}\left(R / I, E_{1}\right) \longrightarrow \operatorname{Hom}_{R}\left(R / I, E_{0}\right) \\
& \longrightarrow \operatorname{Hom}_{R}(R / I, M) \longrightarrow 0
\end{aligned}
$$

is exact and each of the $\operatorname{Hom}_{R}\left(R / I, E_{i}\right)$ is an injective right $R / I$-module since $\operatorname{Ext}_{R / I}^{1}\left(-, \operatorname{Hom}_{R}(R / I, E)\right) \cong \operatorname{Ext}_{R}^{1}(-, E)=0$ by [14, p. 258, 9.21] for any injective right $R$-module $E$. Let $\bar{E}$ be any injective right $R / I$-module. Then $\bar{E}$ is an injective right $R$-module, and so $\operatorname{Hom}_{R / I}\left(\bar{E}, \operatorname{Hom}_{R}(R / I, \mathbb{E})\right) \cong \operatorname{Hom}_{R}(\bar{E}, \mathbb{E})$ is exact and $\operatorname{Ext}_{R / I}^{i}\left(\bar{E}, \operatorname{Hom}_{R}(R / I, M)\right) \cong \operatorname{Ext}_{R}^{i}(\bar{E}, M)=0$ by [14, p. 258, 9.21] for all $i \geq 1$. It follows that $\operatorname{Hom}_{R}(R / I, M)$ is a $\mathrm{G}$-injective right $R / I$-module.

Proposition 3.6. Let $(R, m)$ be a local Noetherian ring $M$ a finitely generated $R$ module and let $\left(a_{1}, \ldots, a_{s}\right)$ be an $M$-regular sequence in $m, \bar{M}=M /\left(a_{1}, \ldots, a_{s}\right) M$. Then $\operatorname{Gpd}_{R} \bar{M}=\operatorname{Gpd}_{R} M+s$.

PROOF. Let $\operatorname{Gpd}_{R} M=n$. We use induction on the finite number $s \geq 1$. If $s=1$, then $\operatorname{Gpd}_{R}\left(M / a_{1} M\right) \leq \operatorname{Gpd}_{R} M+1$ since $0 \rightarrow M \rightarrow^{a_{1}} M \rightarrow M / a_{1} M \rightarrow 0$ is exact. Consider the exact sequence

$$
\operatorname{Ext}_{R}^{n}(M, Q) \longrightarrow \operatorname{Ext}_{R}^{n}(M, Q) \longrightarrow \operatorname{Ext}_{R}^{n+1}\left(M / a_{1} M, Q\right) \longrightarrow 0 .
$$

If $\operatorname{Ext}_{R}^{n}(M, Q) \neq 0$ for some projective $R$-module $Q$, then $\operatorname{Ext}_{R}^{n}(M, R) \neq 0$ since $R$ is Noetherian and $M$ is finitely generated, and hence $\operatorname{Ext}_{R}^{n+1}\left(M / a_{1} M, R\right) \neq 0$ by Nakayama's lemma, which implies that $\operatorname{Gpd}_{R}\left(M / a_{1} M\right)=\operatorname{Gpd}_{R} M+1$. If $s>1$, then

$$
0 \longrightarrow M /\left(a_{1}, \ldots, a_{s-1}\right) M \stackrel{a_{s}}{\longrightarrow} M /\left(a_{1}, \ldots, a_{s-1}\right) M \longrightarrow \bar{M} \longrightarrow 0
$$

is exact and by induction we see that $\operatorname{Gpd}_{R} \bar{M} \leq \operatorname{Gpd}_{R} M+s$. Consider the exact sequence

$$
\begin{aligned}
\operatorname{Ext}_{R}^{n+s-1}\left(M /\left(a_{1}, \ldots, a_{s-1}\right) M, Q\right) & \longrightarrow \operatorname{Ext}_{R}^{n+s-1}\left(M /\left(a_{1}, \ldots, a_{s-1}\right) M, Q\right) \\
& \longrightarrow \operatorname{Ext}_{R}^{n+s}(\bar{M}, Q) \longrightarrow 0 .
\end{aligned}
$$

If $\operatorname{Ext}_{R}^{n+s-1}\left(M /\left(a_{1}, \ldots, a_{s-1}\right) M, Q\right) \neq 0$ for some projective $R$-module $Q$, then $\operatorname{Ext}_{R}^{n+s}(\bar{M}, R) \neq 0$ by Nakayama's lemma. Hence $\operatorname{Gpd}_{R} \bar{M}=\operatorname{Gpd}_{R} M+s$.

By the flat and projective dimensions of a homomorphism of rings $\varphi: R \rightarrow S$ we understand the flat and projective dimensions of $S$ over $R$ respectively; in particular, we say that $\varphi$ is (faithfully) flat if it makes $S$ is a (faithfully) flat $R$-module. We call $\varphi$ finite if it makes $S$ a finite $R$-module. 
Proposition 3.7. Let $R$ and $S$ be rings and let $\varphi: R \rightarrow S$ be of finite projective dimension. If $M$ is a G-projective left $R$-module, then $S \otimes_{R} M$ is a G-projective left S-module.

Proof. Let $P$ be any projective left $R$-module. Then $\operatorname{Ext}_{S}^{1}\left(S \otimes_{R} P,-\right) \cong$ $\operatorname{Hom}_{R}\left(P, \operatorname{Ext}_{S}^{1}(S,-)\right)=0$ by [14, p. 258, 9.20], and so $S \otimes_{R} P$ is a projective left $S$-module. Since $M$ is G-projective, there exists a complete projective resolution $\mathbb{P}: \cdots \rightarrow P_{1} \rightarrow P_{0} \rightarrow P^{0} \rightarrow P^{1} \rightarrow \cdots$ with $M \cong \operatorname{Im}\left(P_{0} \rightarrow P^{0}\right)$. We use induction on the finite number $\operatorname{pd}_{R} S$. If $\operatorname{pd}_{R} S=0$, then $S$ is projective, and $S \otimes_{R} \mathbb{P}$ is exact. Assume $\operatorname{pd}_{R} S \geq 1$. Let $0 \rightarrow K \rightarrow P \rightarrow S \rightarrow 0$ be a projective resolution of $S$ with $\operatorname{pd}_{R} K=\operatorname{pd}_{R} S-1$. Then

$$
0 \rightarrow K \otimes_{R} P_{i} \rightarrow P \otimes_{R} P_{i} \rightarrow S \otimes_{R} P_{i} \rightarrow 0
$$

and

$$
0 \rightarrow K \otimes_{R} P^{i} \rightarrow P \otimes_{R} P^{i} \rightarrow S \otimes_{R} P^{i} \rightarrow 0
$$

are exact for $i=0,1, \ldots$ Thus $0 \rightarrow K \otimes_{R} \mathbb{P} \rightarrow P \otimes_{R} \mathbb{P} \rightarrow S \otimes_{R} \mathbb{P} \rightarrow 0$ is exact, which gives that

$$
S \otimes_{R} \mathbb{P}: \cdots \longrightarrow S \otimes_{R} P_{1} \longrightarrow S \otimes_{R} P_{0} \longrightarrow S \otimes_{R} P^{0} \longrightarrow S \otimes_{R} P^{1} \longrightarrow \cdots
$$

is exact by induction such that $S \otimes_{R} M \cong \operatorname{Im}\left(S \otimes_{R} P_{0} \rightarrow S \otimes_{R} P^{0}\right)$ and $S \otimes_{R} P_{i}$, $S \otimes_{R} P^{i}$ are projective left $S$-modules for $i=0,1, \ldots$ Let $\bar{Q}$ be any projective left $S$-module. Then $\operatorname{pd}_{R} \bar{Q}$ is finite, and so $\operatorname{Hom}_{S}\left(S \otimes_{R} \mathbb{P}, \bar{Q}\right) \cong \operatorname{Hom}_{R}(\mathbb{P}, \bar{Q})$ is exact. Thus $S \otimes_{R} M$ is a G-projective left $S$-module.

PROPOSITION 3.8. Let $R$ and $S$ be rings and let $\varphi: R \rightarrow S$ be of finite flat dimension. If $M$ is a $G$-flat right $R$-module, then $M \otimes_{R} S$ is a $G$-flat right $S$-module.

Proof. Since $M$ is G-flat, there exists a complete flat resolution $\mathbb{F}: \cdots \rightarrow F_{1} \rightarrow$ $F_{0} \rightarrow F^{0} \rightarrow F^{1} \rightarrow \cdots$ with $M \cong \operatorname{Im}\left(F_{0} \rightarrow F^{0}\right)$. Then

$\mathbb{F} \otimes_{R} S: \cdots \longrightarrow F_{1} \otimes_{R} S \longrightarrow F_{0} \otimes_{R} S \longrightarrow F^{0} \otimes_{R} S \longrightarrow F^{1} \otimes_{R} S \longrightarrow \cdots$

is exact by an argument like the proof of Proposition 3.7 such that $M \otimes_{R} S \cong$ $\operatorname{Im}\left(F_{0} \otimes_{R} S \rightarrow F^{0} \otimes_{R} S\right)$ and $F_{i} \otimes_{R} S$ and $F^{i} \otimes_{R} S$ are flat right $S$-modules by [7, p. 43, Exercise 9] for $i=0,1, \ldots$ Let $\bar{I}$ be any injective left $S$-module and let $H$ be any left $R$-module and $\mathbb{P}$ be a projective resolution of $H$. Then $S \otimes_{R} \mathbb{P}$ is a projective resolution of $S \otimes_{R} H$ over $S$. Thus

$$
0=\operatorname{Ext}_{S}^{1}\left(S \otimes_{R} H, \bar{I}\right)=\mathrm{H}^{1}\left(\operatorname{Hom}_{S}\left(S \otimes_{R} \mathbb{P}, \bar{I}\right)\right) \cong \mathrm{H}^{1}\left(\operatorname{Hom}_{R}(\mathbb{P}, \bar{I})\right)=\operatorname{Ext}_{R}^{1}(H, \bar{I})
$$

and hence $\bar{I}$ is an injective left $R$-module. So $\mathbb{F} \otimes_{R} S \otimes_{S} \bar{I} \cong \mathbb{F} \otimes_{R} \bar{I}$ is exact, which implies that $M \otimes_{R} S$ is a G-flat right $S$-module.

Proposition 3.9. Let $R$ and $S$ be rings and let $\varphi: R \rightarrow S$ be of finite projective dimension. If $M$ is a $G$-injective left $R$-module, then $\operatorname{Hom}_{R}(S, M)$ is a $G$-injective left S-module. 
Proof. Let $E$ be any injective left $R$-module. Then it follows from [14, p. 258, 9.21] that $\operatorname{Ext}_{S}^{1}\left(-\operatorname{Hom}_{R}(S, E)\right) \cong \operatorname{Ext}_{R}^{1}(-, E)=0$, and so $\operatorname{Hom}_{R}(S, E)$ is an injective left $S$-module. Since $M$ is G-injective, there is a complete injective resolution $\mathbb{E}: \cdots \rightarrow E_{1} \rightarrow E_{0} \rightarrow E^{0} \rightarrow E^{1} \rightarrow \cdots$ with $M \cong \operatorname{Im}\left(E_{0} \rightarrow E^{0}\right)$. Then

$$
\begin{aligned}
\operatorname{Hom}_{R}(S, \mathbb{E}): \cdots \longrightarrow \operatorname{Hom}_{R}\left(S, E_{1}\right) & \longrightarrow \operatorname{Hom}_{R}\left(S, E_{0}\right) \longrightarrow \operatorname{Hom}_{R}\left(S, E^{0}\right) \\
& \longrightarrow \operatorname{Hom}_{R}\left(S, E^{1}\right) \longrightarrow \cdots
\end{aligned}
$$

is exact by analogy with the proof of Proposition 3.7 such that $\operatorname{Hom}_{R}(S, M) \cong$ $\operatorname{Im}\left(\operatorname{Hom}_{R}\left(S, E_{0}\right) \rightarrow \operatorname{Hom}_{R}\left(S, E^{0}\right)\right)$ and $\operatorname{Hom}_{R}\left(S, E_{i}\right), \operatorname{Hom}_{R}\left(S, E^{i}\right)$ are injective left $S$-modules for each $i$. Let $\bar{J}$ be any injective left $S$-module. Then $\bar{J}$ is an injective left $R$-module, and $\operatorname{son}_{S}\left(\bar{J}, \operatorname{Hom}_{R}(S, \mathbb{E})\right) \cong \operatorname{Hom}_{R}(\bar{J}, \mathbb{E})$ is exact. Thus $\operatorname{Hom}_{R}(S, M)$ is a G-injective left $S$-module.

\section{References}

[1] F. W. Anderson and K. R. Fuller, Rings and Categories of Modules (Springer, Berlin, 1992).

[2] M. Auslander and M. Bridger, 'Stable module theory', Mem. Amer. Math. Soc. 94 (1969).

[3] L. W. Christensen, Gorenstein Dimensions (Springer, Berlin, 2000).

[4] L. W. Christensen, A. Frankild and H. Holm, 'On Gorenstein projective, injective and flat dimensions - a functorial description with applications', J. Algebra 302 (2006), 231-279.

[5] N. Q. Ding and J. L. Chen, 'Coherent ring with finite self-FP-injective dimension', Comm. Algebra 24 (1996), 2963-2980.

[6] E. E. Enochs and O. M. G. Jenda, 'Gorenstein injective and projective modules', Math. Z. 220 (1995), 611-633.

[7] Relative Homological Algebra (Walter de Gruyter, Berlin, 2000).

[8] E. E. Enochs and J. A. López-Ramos, 'Kaplansky classes', Rend. Sem. Math. Univ. Padova 107 (2002), 67-79.

[9] H. Holm, 'Gorenstein homological dimensions', J. Pure Appl. Algebra 189 (2004), 167-193.

[10] F. Kasch, Modules and Rings (Academic Press, London, 1981).

[11] T. Y. Lam, Lecture on Modules and Rings (Springer, Berlin, 1999).

[12] L. X. Mao and N. Q. Ding, 'Cotorsion modules and relative pure-injectivity', J. Aust. Math. Soc. 81 (2006), 225-243.

[13] M. S. Osborne, Basic Homological Algebra (Springer, Berlin, 2000).

[14] J. J. Rotman, An Introductions to Homological Algebra (Academic Press, New York, 1979).

[15] J. Trlifaj, 'Ext and inverse limits', Illinois J. Math. 47 (2003), 529-538.

[16] J. Xu, Flat Covers of Modules, Lecture Notes in Mathematics, 1634 (Springer, Berlin, 1996).

[17] X. Y. Yang and Z. K. Liu, ' $\Omega$-Gorenstein projective, injective and flat modules', Algebra Colloq., to appear.

ZHONGKUI LIU, Department of Mathematics, Northwest Normal University, Lanzhou 730070, PR China

e-mail: liuzk@nwnu.edu.cn

XIAOYAN YANG, Department of Mathematics, Northwest Normal University, Lanzhou 730070, PR China

e-mail: yxy800218@163.com 